LA-7928-MS

Informal Report

Current Redistribution in Cables Made of Insulated, Soldered, or Oxidized Strands 
LA-7928-MS

Informal Report

UC-20b

Issued: July 1979

\title{
Current Redistribution in Cables Made of Insulated, Soldered, or Oxidized Strands
}

\author{
B. Turck
}

*Visiting staff memiber. Centre d'etudes Nucleaires de Saclay, BPnㅁ2, GIF-sur-yvette (91190) FRANCE.
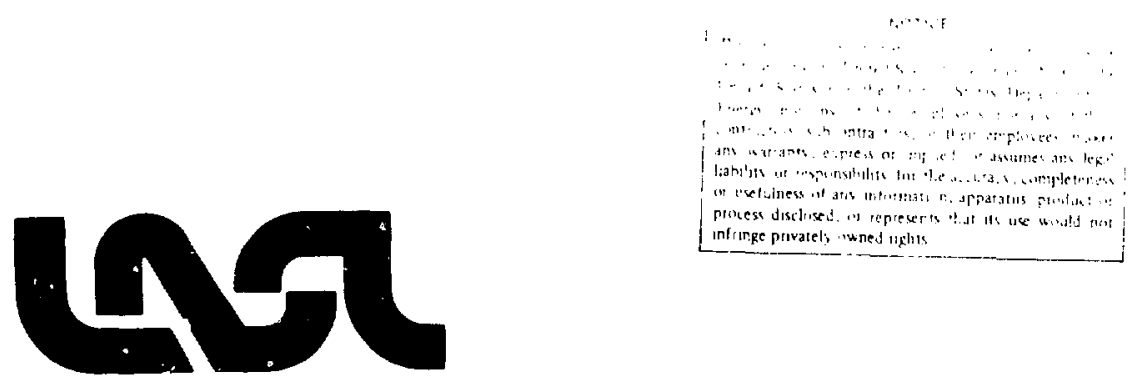


\title{
CURRENT REDISTRIBUTION IN CABLES MADE OF INSULATED, SOLDERED, OR OXIDIZED STRANDS
}

by

B. Turck

\begin{abstract}
Current redistributions are compared in cables made of insulated strands, soldered, or oxidized strands and insulated strands with periodic joints. After discussing the different current redistributions in the cases of a rapidly changing current and a dc current, several particular situations are investigated: what happens if a strand is broken, or if a local normal zone appears that does not affect all the strands equally, the detection of this normal zone, and the influence of short circuits between strands.
\end{abstract}

\section{INTRODUCTION}

A lot of magnet projects involving the use of high-current conductors require cables or braids made by twisting or interweaving a number of strands. These strands are usually soldered in the case of magnets supplied with a dc current or a slowly varying current (dipoles for an accelerator). Besides, for very large current carrying conductors subjected to rapidly varying magnetic fields, such as in the toroidal or poloidal field coils of a tokomak or in an ac power generator, it is generally proposed that the strands are electrically insulated from each other to reduce the ac losses to an acceptable level. The aim here is to compare the current redistributions as functions of time and space in three different kinds of conductors under various particular conditions. 


\section{A. General}

It has already been shown ${ }^{1,2}$ that in a coil supplied with an ac current, or during the transient period when a constant current is suddenly applied, the currents share between strands so that the actual net flux enclosed by each strand is equal. To compensate for any discrepancy between these fluxes, circulating currents are induced from strand to strand to satisfy the above condition. In other words, a linear circuit theory shows that the current distributions is given by expressions involving the quantities $L_{i}-M_{i j}$, where $L_{i}$ is the self inductance of strand $i$ and $M_{i j}$ is the mutual inductance between strand $i$ and strand $j$. Besides, the sharing is not given by the absolute values of these quantities but only by the differences between them, which are usually a small part of $L_{i}-M_{i j}$, except if the transposition is not perfect, or if a few strands are not supplied with current for any reason. If the magnet is supplied by a dc current, this particular distribution persists during a time span as a function of a series of time constants, of the order of $\mathrm{L}_{i}-\mathrm{M}_{\mathrm{ij}} / \mathrm{r}_{\mathrm{i}}$, where $\mathrm{r}_{\mathrm{i}}$ is a typical resistance in series with the stand $i$. Then the current distribution tends to an equilibrium as a function of the combined effect of all the series and parallel resistances. In the particular and useful case where the syster is well represented by series resistance $r_{i}$ in each strand, the final current in the strand $i$ is exactly inversely proportional to the series resistances $r_{i}$. Note that the resistance in series with each strand is the sum of the resistance of any joint and the resistance of the transfer length at the solder joint to the current lead. This is given by

$$
\mathrm{R}_{\mathrm{S}}=\sqrt{\mathrm{R}_{\mathrm{L}} / \mathrm{G}_{\mathrm{t}}}
$$

where $R_{L}$ is the longitudinal resistance per unit length of the copper of the current lead and $G_{t}$ is the transverse conductance per unit length and includes the copper of the current lead, the soldering, and the transverse conductance of the comporite. These resistances are usually very smill (in the range of $10^{-9} \Omega$ to $10^{-7} \Omega$ ) and can be different by a factor of at least 2 . As a result, the current distribution can be quite unequal. 
B. How To Achieve An Equal Current Sharing

All the quantitive $L_{i}-M_{i j}$ can be measuied independently as shown in Fig. 1. The conductor $i$ is supplied with a current at a frequency w/2\%. The current $I$ is measured by means of the shunt resistor, $R$. The other conductors are not connected on one end. The voltage between strand $i$ and strand $j$ appears to be simply $\left(\mathrm{L}_{\mathrm{i}}-\mathrm{M}_{\mathrm{ij}}\right)$... Linear current theory shows that small inductances can be put in series with each strand to make up for the discrepancies among the currents. This is rather easy to do in a cable made of small number of strands but is almost inpractical if the number of strands is large. Moreover, this correction is effective only in a magnet supplied with an ac current. In a magnet supplied with a de current, the fact that the current tends towards an equilibrium after the lapse of a period of the order of a few time constants suggests a second way to equalize currents. A small resistance of $10^{-7}$ to $10^{-6}$... can be put in series with each strand. Tire effect is both to reduce the time constant and to balance the currents at the cost of some heat generation. Both techniques were used successfully during the test of the pulsed magnet MOBY at Saclay. ${ }^{1}$

\section{CURRENT SHARTNG BETWEEN STRANDS IN A SOLDERED CABLE OR AN OXIDIZED}

\section{STRAND CABLE}

The case is considered for a transverse conductance layer of typically $10^{4}(\varsigma r \mathrm{~m})^{-1}$ existing between strands. This conductance enables local redistribution of current. The influence of such a transverse conductance has

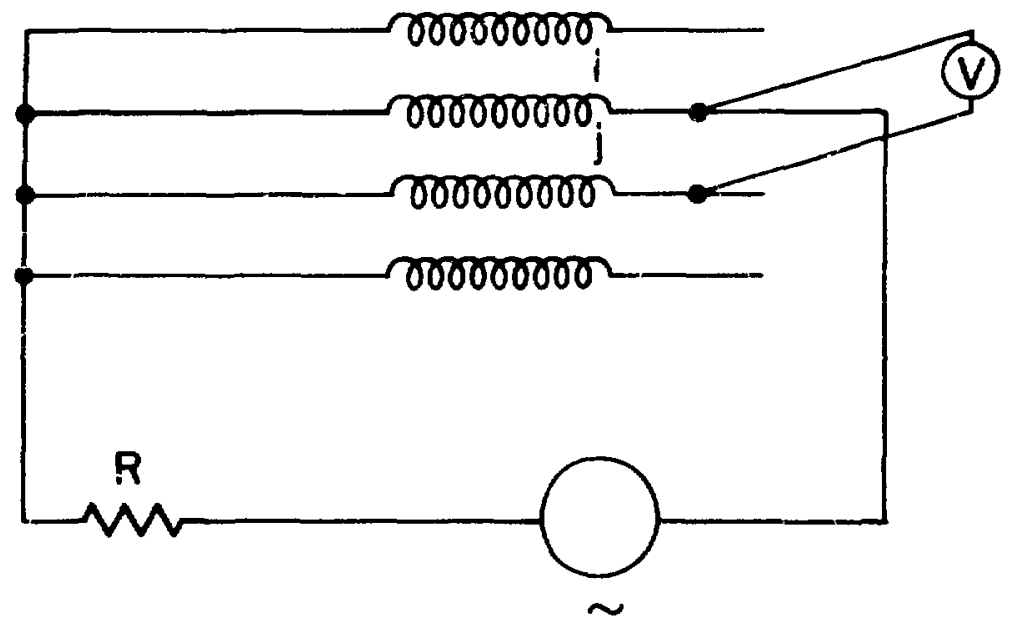

Fig. 1. Measurement of the difference $\mathrm{L}_{i}-\mathrm{M}_{i j} \cdot$ 
been studied in large detail in a previous publication. ${ }^{2}$ The main results are the following:

When the current is turned on, the initial distribution does not depend at all on the transverse conductance. It depends only on the quality of transposition achieved between strands as in Sec. II, that is, on the different values of self and the mutual inductances. It is usually difficult to know if each wire carries the same current. Then, in the vicinity of the terminals, another distribution takes place. The currents tend to local values in inverse proportions to the series resistance at the joint with the current leads, and this is after a time equal for instance to the time constant $\left(1_{1}+1_{2}-2 m\right) / 4 r^{2} G$ in a two-strand cable, where

$1_{1}, 1_{2}$, are the self inductances of strands 1 and 2 , per unit length $m$ is the mutual inductance between the strands, per unit length

$r$ is the series resistance, and

$G$ is the transverse conductance per unit length of conductor.

In an oxidized stranded cable without extra series resistance, other than the solderings to the current leads, this time constant can be of the order of $10^{4} \mathrm{~s}$, whereas, in a soldered cable it can be of the order of $1 \mathrm{~s}$. As a result, in a magnet supplied with an ac current, the current distribution is practically given only by transposition effects. This is at variance from a magnet supplied with a dc current. In such a case, the current distribution produced by the serıes resistance near the terminals propagates with a diffusion velocity such that after a time span proportional to the square of the length of the cable, each strand carries the same current along its entire length.

The diffusivity is $\mathrm{D}_{\mathrm{m}}=$ 1 in a two-strand cable. $\mathrm{G}\left(\mathrm{l}_{1}+\mathrm{l}_{2}-2 \mathrm{~m}\right)$

In a homogeneous medium, this diffusivity is equivalent to the usual magnetic diffusivity $D_{m}=\rho \perp / \mu O$, where $\rho \perp$ is an equivalent transverse resistivity between strands.

The main advantage of a partially insulated stranded cable is that current sharing between strands is possible because of the transverse conductance. This is considered in the following section. 
IV. INFLUENCE OF A RESISTANCE IN SERIES WITH ONLY A FEW CABLE STRANDS

A local series resistance can occur in three particular cases of inter-

est. These are

a. One or a few strands happen to he broken.

b. A finite resistance exists in a bad wire caused by a local interruption of the superconductor. Cold soldering of a joint is a good example of this case. The particular situation where a normal zone appears locally is discussed separately because of the specific behaviour of the changing resistance.

c. The current becomes locally critical in only one or a few strands.

\section{A. Insulated Stranded Cable}

1. If a strand is broken $(R=x)$, the strand obviously does not carry any current. Morecver, in a cable made with more than three strands, the perfect transposition cannot exist any longer between the good strands. As a result and as stated above, the current distribution is definitely unequal in a cable supplied with an ac current.

2. If only the superconductor is broken, the resistance remains finite with copper matrix, joint solder of any $k$ ind, etc., the current does not increase any more in the faulty wire after the initial distribution; and the total current is almost entirely carried by the other wires. The time to reach this new distribution is of the order of $\frac{2(L-M)}{2 r+R}$ in a two-strand cable. In a triplet, the mutual inductances are theoretically equal; the same is true for the selt inductances. Inere are two time conscancs oí lite utuer uf $\frac{L-M}{r}$ and $\frac{3(\mathrm{~L}-\mathrm{M})}{3 r+2 \mathrm{R}}$. See Fig. 2 .

3. If the current reaches its critical value in one strand whereas it remains far from critical in the other strands, a series resistance is deve1oped locally caused by the resistive aspect of the critical state. In a magnet supplied with an ac current there is little hope to observe a redistribution between strands before the total quenching of the bad strand. If the current sweep rate is low enough, almost a constant current, then a redistribution to the other strands can be expected because of the voltage developed across the resistive zone. The time constant of the circulating current is very large, since only very small series resistances are involved. 


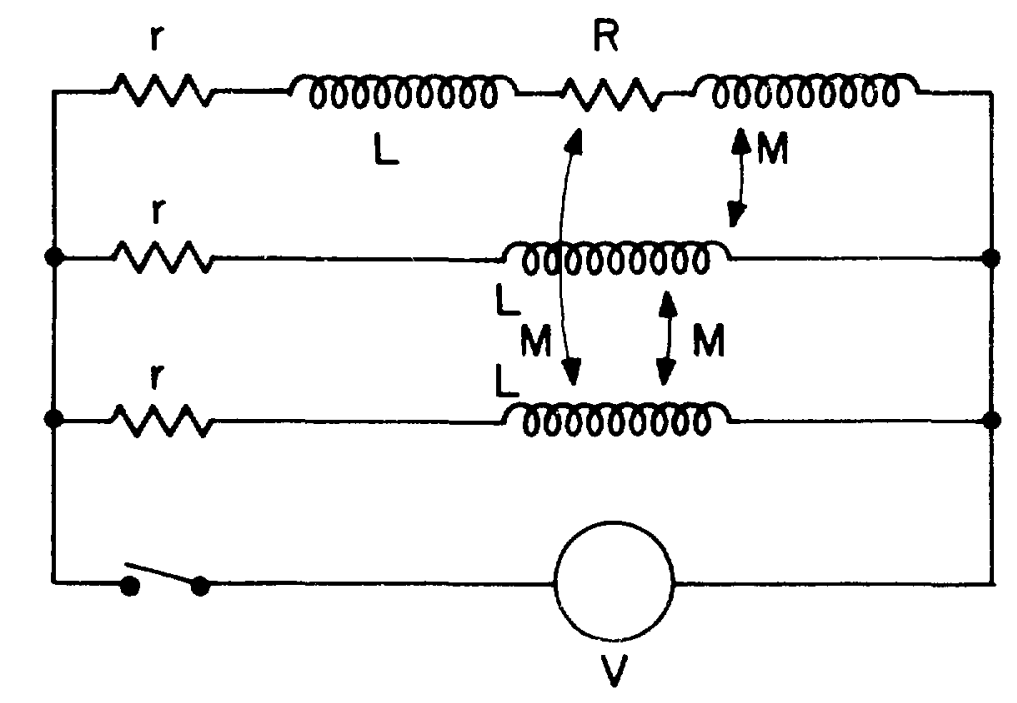

Fig. 2. Simulation of a resistive zone in a strand.

As a rule, a cable is calculated to be cryostable at an operating current substantially less than critical. Therefore, a strand carrying a critical current is expected to be unstable and may go normal locally. Nevertheless, if there is enough copper to stabilize the strand at the critical current, the current can continue to increase at a lower rate in the copper of that strand simultaneously with a progressively increasing redistribution to the other strands. The current distribution tends toward an equilibrium, with a current in that strand a little in excess of the critical current.

\section{B. Soldered Cable}

For the sake of simplification, consider only the case of a two-strand cable.

1. Theoretical Case. Just after the main current has been supplied, the sharing is only a function of the self and mutual inductances. Then, very soon, in the vicinity of the resistance or the break the current decreases in the corresponding strand to zero or a finite value if the series resistances are not negligible with respect to the total transverse resistance between the two strands. This takes place with a time constant equal to $\frac{l_{1}-l_{2}-2 \mathrm{~m}}{R^{2} G}$ if $R \gg r .^{2}$ See Fig. 3. Simultaneously, a redistribution all along the cable propagates with a diffusion velocity $D_{m}=\frac{\cdot 1}{G\left(\ell_{1}-\ell_{2}-2 m\right)}$. Thus, after a time which can be very long, the faulty wire is without current or nearly so, as determined by the relative values of the series and transverse resistances. 


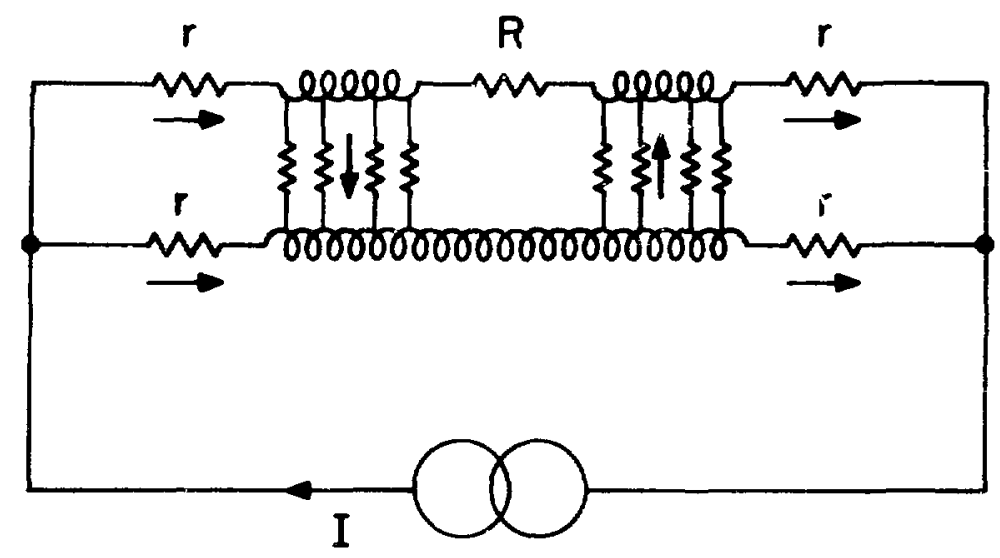

Fig. 3. Cable with a transverse conductance between strands.

Practically all the current is carried by the other wires. For a magnet supplied by an ac current, the current can be well distributed between every strand except in the vicinity of the break because of the large time for the diffusion process.

2. Practical Case. To understand the phenomenon, two cases are investigated separately.

a. Negligible series resistances at the joints (see Fig. 4). The current decreases towards zero in the faulty wire and increases in the others until a new situation exists. When, in any of the good strands, the current becomes locally critical at a point such as $A$ of conductor 1 in Fig. 4, a small voltage, $V$, appears. This potential enables current to flow across the transverse conductance from the faulty strand to the good ones. Thus, a current can flow in the broken strand in the region where one of the others is in the critical state. This occurs only to prevent the sirand in the critical state from becoming over critical. This situation is, of course, stable, sinse any tendency for the conductor to carry a current larger than critical results in a higher voltage and a transfer of current to the broken conductor. From Fig. 4, the equilibrium for two strands is seen to be

$$
\begin{aligned}
& V=R_{t}\left(I-i_{c}\right) \text { and } \\
& V \simeq R_{e} i_{c}
\end{aligned}
$$




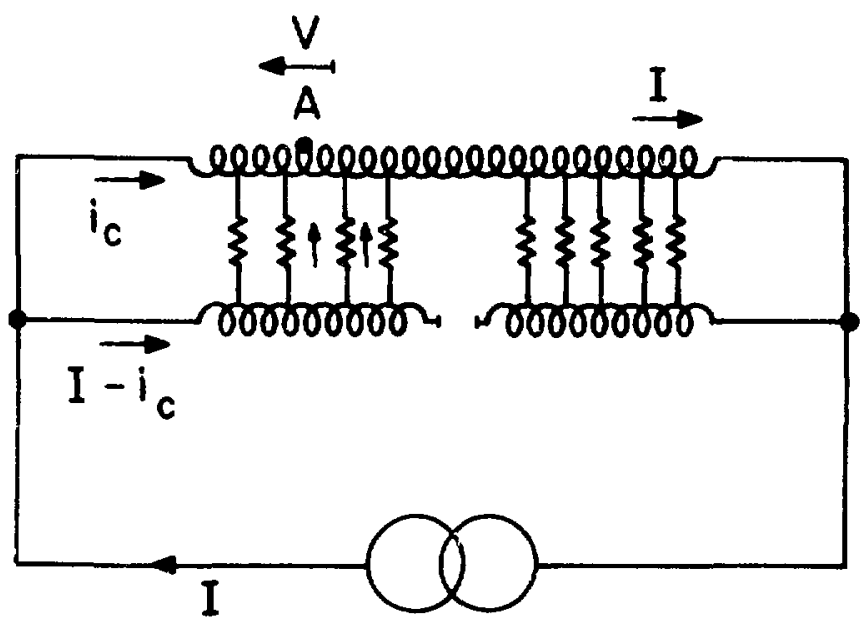

$\mathbf{a}$

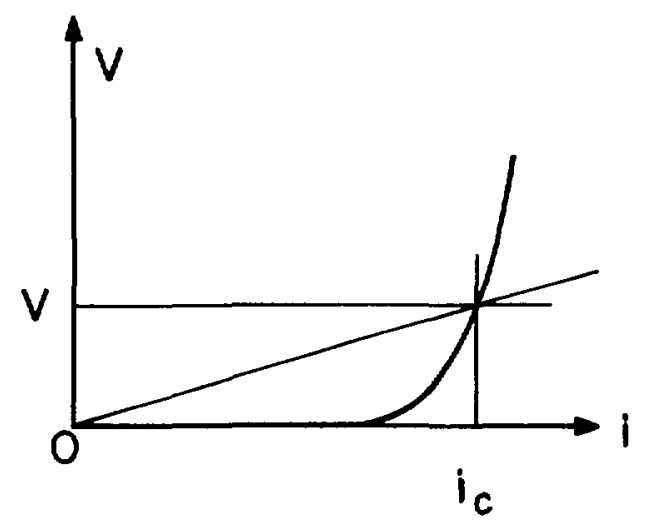

b

Fig. 4. a) A broken strand in an oxidized strand cable.

b) The critical state.

where $R_{t}$ is the involved transverse resistance and $R_{e}$ is the equivalent resistance of the conductor in the critical state.

From these equations the value of the equivalent resistance, $R_{e}$, is

$$
\frac{I}{I c}=\frac{R_{t}+R e}{R_{t}} .
$$

Apparently there is no special problem except if the break is located either in a region of high field producing the critical state or just at the input of the inner layer of the coil on the high field side. If the break is partly reduced by any kind of soldering and since the resistance $R$ is of finite value, then the situation is even far better.

b. Nonnegligible series resistances at the joints or at the ends. See Fig. 5. After the transient period is over, a dc current, $i_{2}$, can flow in the broken strand, due to the potential srop, $V_{1}$, across the resistance of the joints, except in the close vicinity of the break. That current is such that the voltage, $v_{3}$, always remains smaller than $v_{1}$. When $r_{1}=r_{2}=r$, the current, $i_{2}$, is smaller than $i_{1}$. The final current distribution can be calculated by witing the equations for Fig. 5b. The four currents are 


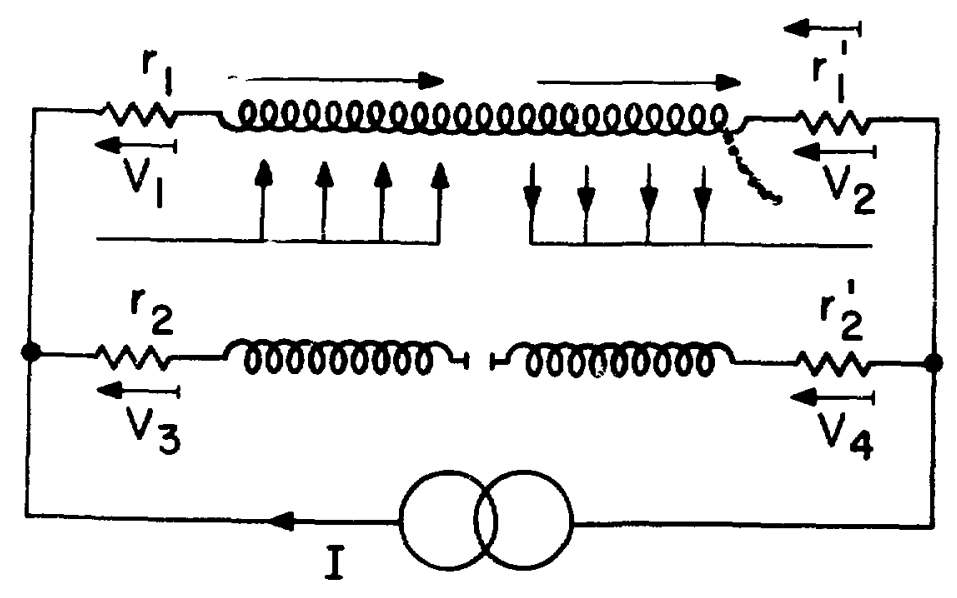

a

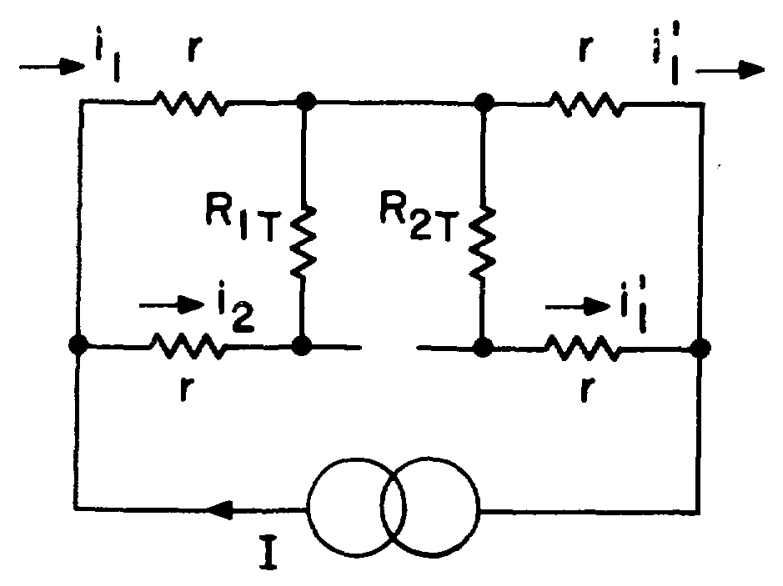

b

Fig. 5. a) Influence of the series resistance on the current sharing. b) Simplified scheme.

$$
\begin{aligned}
& i_{1}=I \frac{r+R_{1 t}}{2 r+R_{1}}, \\
& i_{2}=I \frac{r}{2 r+R_{1 t}}, \\
& i^{\prime}{ }_{1}=I \frac{r+R_{2 t}}{2 r+R_{2 t}}, \text { and } \\
& i^{\prime}{ }_{2}=I \frac{r}{2 r+R_{2 t}} .
\end{aligned}
$$

Thus, the series resistances at the joints wake possible the transfer of current in the faulty strand. If the broken strand has been soldered to reduce the extra series resistance, $R$, to a rather small value, then the current carrying capacity of the faulty strand is increased. In practice, in a coil where the conductor is long enough to make the transverse resistances, $R_{1 t}$ and $R_{2 t}$, smaller than the resistances, $r$, at the joints, a noticeable 
current can flow in each wire. P. Genevey ${ }^{3}$ has already observed that disconnecting two strands of an oxidized triplet cable on the low field side of a coil is not at all catastrophic. In his experiment, the critical current nf the coil was indeed reached.

\section{QUENCHING OF ONLY A FEW STRANDS IN AN INSULATED STRAND CABLE}

A local energy release may give rice to a normal zone involving only a few strands. For example, in a typical case, if the heating is caused by friction of the cable sliding on a spacer located on one side, only half the strands can go normal. The resistance of a normal zone is changing with time. It can either increase and then decrease for recovery or continuously increase and quench.

\section{A. Recovery}

Recovery occurs if the conductor is cryostable or if the process of current redistribution to the other strands is sc fast that the current decreases in the normal conductor to a value such that the strand becomes stable. A typical resistance change is shown in Fig. 6 with a time span in the range of 0.1 to $1 \mathrm{~s}$. As shown above, as soon as a resistance, $R$, appears in a strand, current is transferred to the other strand with a time constant $\mathrm{T}_{\text {em }}$. Thus, differenct situations can occur based mainly on the ratio of the two time constants -- the electromagnetic time constant, $T_{\text {em, }}$ and the recovery time constant, $\mathrm{T}_{\mathrm{r}}$.

\section{The Case of Large Electromagnetic Time Constant $\mathrm{T}_{\mathrm{em}} \geqslant \mathrm{T}_{\mathrm{r}}$. This} is the case for large coils with $\mathrm{T}_{\text {em }}-100$ to $1000 \mathrm{~s}$. The different strands benave independently, and the current sharing between strands cannot take place during the recovery time of $\sim 1 s$.

\section{The Case of Small Electromagnetic Time Constant $T_{e m} \ll T_{r}$. This} is the case for small coils with $T_{\text {em }} \sim 0.01$ to $0.1 \mathrm{~s}$. The current decreases in the normal strand to a value, $I \frac{r}{2 r+R_{1}}$, where $R_{1}$ is the resistance of the normal zone of strand 1 . This value is almost zero. In the other strand the current becomes equal to $I \frac{r}{2 r+R_{1}}$, which is almost the total current $I$. Now, the second strand which carries the total current, $I$, is likely not to be cryostable for that current. If, in turn, it goes normal with a resistance $R_{2}$, it can be overheated by the joule effect, unless the new electromagnetic constant related to the resistance, $R_{2}$, is so small that current sharing to 


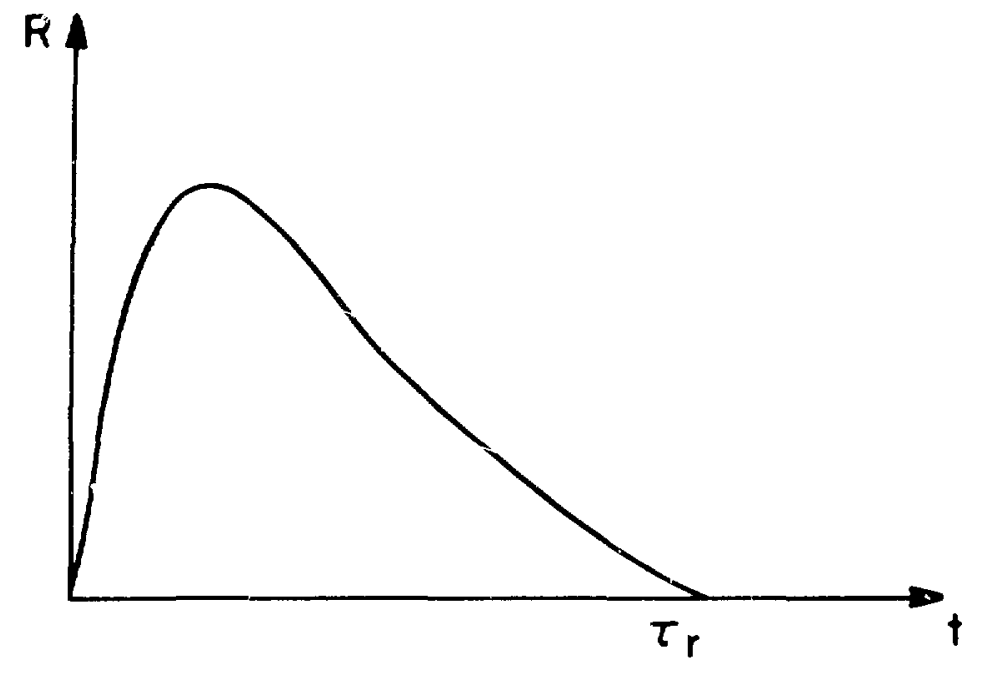

Fig. 6. Typical resistance of a recovering normal
zone versus time.

the first strand prevents the second strand from undergoing a full quenching and eventually melting. In the meantime, the first strand may or may not have recovered; and the process can stop or continue in an oscillating mode. If strand 1 has not recovered, then the currents share according to values inversely proportional to the resistances, $R_{1}$ and $R_{2}$. In the whole process, $R_{2}$ can be expected to be larger than $R_{1}$, which results in a current $i_{1}$, larger than $i_{2}$. Conductor 1 , in turn, may not be cryostable. Damages or at least oscillations are likely.

3. The Case of The Two Time Constants of the Same Order of Magnitude $T$ em $=\mathrm{T}_{\mathrm{r}}$. At first, when the resistance, $\mathrm{R}_{1}$, appears, there is a current sharing between the two strands in a time of the order of $T_{\text {em }}$ Then, if the recovery is rather fast, the electromagnetic time constant can increase to large values for $R \rightarrow 0$, only limited by the series resistances at the joints. It is easily understo 1 that the redistribution towards an equal current sharing can take a very long time during which the second strand carries the total current and may not be cryostable.

In sumary, large electrcmagnetic time constants are better and safer to let every strand behave independently, although a short electromagnetic time constant may bring sharing and help recovery in some particular experimental situations. 


\section{B. Quenching}

The normal zone in the strand propagates, which results in a decrease of the electromagnetic time constant and some current transfer to the other strarid. Nevertheless, the main problem is the detection of the appearance of a normal zone when it does not affect all the strands equally. That has been clearly seen in the experiments detailed in Ref. 4.

VI. DETECTION OF A NORMAL ZONE IN A FULLY INSULATED STRAND CABLE

When the whole cable goes normal locally, i.e., all the strands over a short length, the detection of the normal zone does not thow any special difficulty. On the contrary, as soon as a resistive zone that affects only a few strands is developed, an inductive voltage in opposite direciion tends to transfer the current to the other strands. Reference 4 shows for the case of a two-strand cable that the overall voltage which can be detected is noticeably reduced. If strand 1 is turned normal with a resistance, $R$, taken here as a constant, then

$$
v \searrow \frac{R I\left(L_{2}-M\right)}{2\left(L_{1}+L_{2}-2 M\right)} e^{-t / \tau} \text {, }
$$

where $I$ is the total current and is the time constant. Next is shown that in a cable made of a large number of strands, when only one strand turns normal, the measured voltage is much smaller than might be expected from the product of the resistance of the normal zone by the current in that strand. To make the calculations easier, consider a cable made by three fully transposed strands. A resistance, $R$, appears in strand 1 . The total current is kept constant. See Fig. 2. The currents are identical in strands 2 and 3 , and

$$
i_{1}=I-2 i_{2}
$$

The voltage in strand 1 is

$$
V=L \frac{d i_{1}}{d t}+2 M \frac{d i_{2}}{d t}+(R+r) i_{1}
$$


and in strands 2 and 3 is

$$
V=M \frac{d i_{1}}{d t}+(L+M) \frac{d i_{2}}{d t}+r i_{2} \cdot
$$

Performing some algebra leads to the time constant

$$
\tau=\frac{3(L-M)}{3 r+2 R}
$$

and to the inirial voltage

$$
V_{0}=I\left(\frac{R}{9}+\frac{r}{3}\right)
$$

For a number of strands greater than 3, the currents in the strands other than 1, which goes normal, are not equal; and the calculations are tedious. They lead to the general conclusion that if only one strand is turning normal, the voltage used to detect the occurence of a normal zone decreased rapidly with the number of strands (a little less rapidly than $n^{-2}$ ). In a three-strand cable the initial voltage is equal to $\mathrm{RI} / 9$ instead of $\mathrm{RI} / 3$ and then decreases exponentialiy with time.

VII. ARE SHORT CIRCUITS BETWEEN INSULATED STRANDS CATASTROPHIC?

In a magnet supplied with a dc current, the currents flow according to the inverse of the series resistences. If they are distributed to insure equipotentials locally, there is no current in the short circuit. But that is most unlikely, and the small current flowing in the short circuit is only limited by the contact resistance between strands. This can usually be of the order of $10^{-3}$ to $10^{-1} \Omega$, which is much larger than the joint resistances. As a result, the current flowing and the power dissipated in the short circuit under de conditions are negligible. Usually, short circuits are dangerous only in magnets supplied with a varying current. 


\section{A. Perfectiy Transposed Cable}

A cable cannot be fully transposed in a magnetic field gradient; however, consider a theoretically perfectly transposed cable. The maximum current which can flow in a short circuit is proportional, at most, to the area of the loop of a half twist pitch length, see Fig. 7. From A to $B$ the net flux is zero. In the loop BCD, the circulation of the electric field is not zero. The current is not only limited by the resistance of the short circuit but mainly by the self and mutual inductance of the strands. The extra loop, $B C D$, results in a difference between the self inductances $L_{1}$ and $L_{2}$ up to points $C$ and $D$. The circulating current is then given by

$$
i_{c}=\frac{I}{2} \quad \frac{\mathrm{L}_{2}-\mathrm{L}_{1}}{\mathrm{~L}_{1}+\mathrm{L}_{2}-2 \mathrm{M}}
$$

when the resistance of the short is negligible. If the difference $\mathrm{L}_{2}-\mathrm{L}_{1}$ is given only by the area of a half twist pitch loop, the current, $i_{c}$, is very small.

When field gradient exists or in an actual coil, $\mathrm{L}_{2}-\mathrm{L}_{1}$ is not so small. The gradient, $\Delta \mathrm{B}$, must be taken into account over the whole length of the cable, and the circulating current may not be negiigible even in a theoretically fully transposed cable.

However, if there is a large number of shorts in the cable, the current loops are smaller, the local values of $(L-M)$ are correspondingly reduced and the short circuit current can be significant.

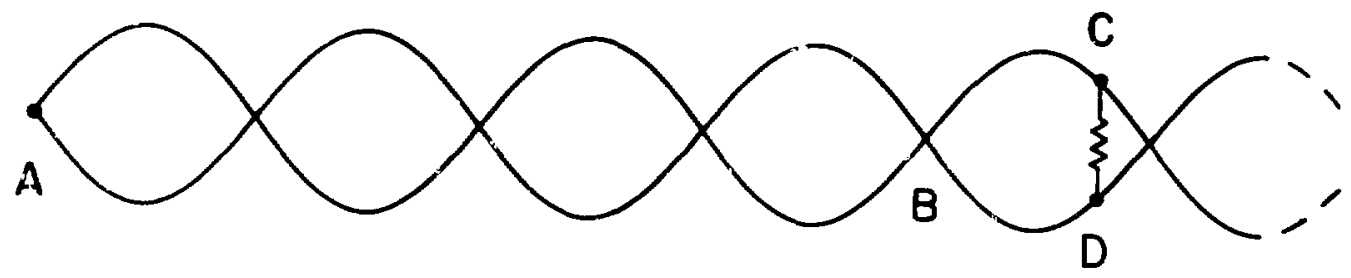

Fig. 7. A short clrcuit in a perfectly transposed cable. 
In an imperfectly trensposed cable, circulating currents flow between strands to keep the net flux equal to zero between every pair of strands. When there is no short circuit between strands, that current returns at the ends. In tite short circuited case, a current can flow through the short circuit to close the loop locally. The actual situation is developed in more detail below.

1. At first, the assumption is made that the imperfections in transposition are equally distributed along the cable, i.e., a regularly repetitive gemetric error due to a deformation of cable. This situation is well represented in Fig. 8 when the short is located just at the middle of the cable. Every twist pitch length exhibits a big loop and a small loop. The enclosed flux of the external field is not zero; thus, a circulating current flows to make the net flux zero. If the short circuit is located just in the middle of the cable at point $A$, the overall current flowing from one strand to the other through the short circuit is zero. Of course, if the short circuit is located elsewhere (point B), the current through it is proportional to the difference of the net flux linked on both sides.

2. A bad winding and a field gradient can result in a transposition imperfection. The circulating current turning at the end makes the overall net flux, but not the local flux, equal to zero. Moreover, in the most likely case shorts are not located right in the middle of the cable, resulting in the existence of $a$ big and a small loop. Thus, a short circuit divides the cable into two regions, one in a low field and the other in a high field. As a result, a current can flow in the short circuit to make the flux in these two

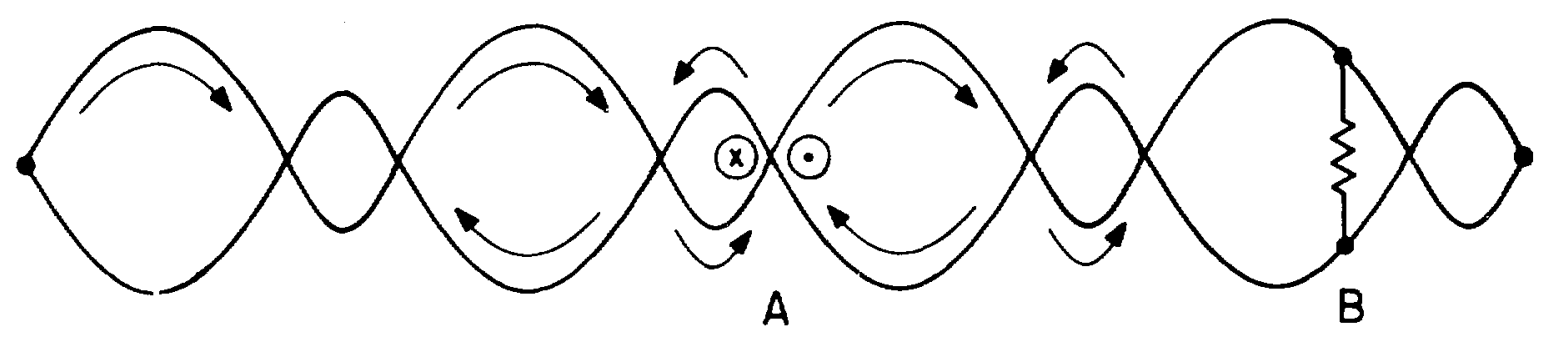

Fig. 8. A short circuit in an imperfectly transposed cable. 
parts, separately equal to zero. Serious effects of short circuits between strands in an imperfectly transposed cable can be expected. They increase against the local net flux.

VII I. AN INTERMEDIATE SITUATION: LOCAL AND PERIODIC JOINTS

Joints may exist periodically along the length of the cable, for instance, at every one or two layers of a coil. This creates localized combinations of series, parallel, and bridged resistances between strands. These joints are investigated further for a three-strand cable. In a two-strand cable, joints are simulated by series and parallel resistances. See Fig. 9.

A. Current Sharing

The initial current distribution function of self and mutual inductances involves the main circulating currents returning at the ends and local circulating current returning across each joint. The two currents depend on tine ratio of the series resistance $r_{L}$, and parallel resistance, $r$.

For a large number of joints, $n$, the overall time constant is of the or ser of $\frac{\mathrm{L}-\mathrm{H}}{\mathrm{nr}}$; and the local time constant is about $\frac{\mathrm{L}-\mathrm{M}}{\mathrm{n} 2\left(\mathrm{r}_{\mathrm{L}}+\mathrm{rt}_{\mathrm{t}}\right)}$. The main result is a noticeable reduction of the effective time constant for current redistribution between strands.

B. Influence of a Local Resistance in One Strand

If one strand is broken or if a normal zone appears locally, then, after a given time and if $\mathbf{r}_{L} \gg \mathbf{r}_{t}$, the other strand carries all the current in the parallel strand connected by the transverse resistances on

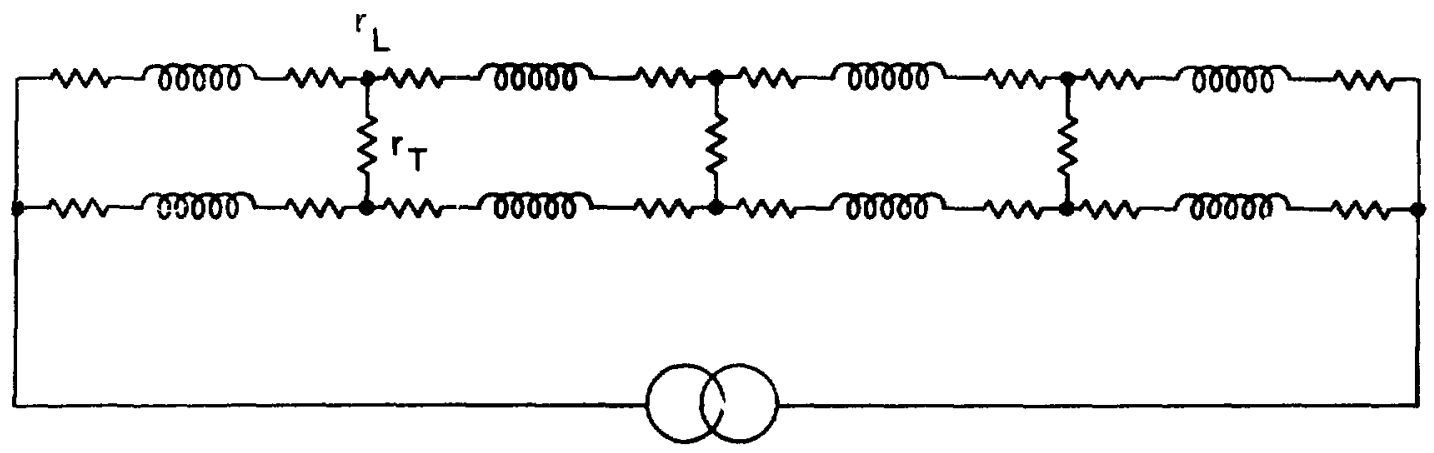

Fig. 9. A cable with perfodically located joints. 
either side of the break or normal zone. When $r_{L}$ and $r_{t}$ are of the same order of magnitude, the other strand carries more current locally but less than in the above case. There is a reaction on the other sections, which decraases with the distance from the additional series resistance due to the break or normal zone.

C. Current Distribution Under DC Conditions

The equilibrium current distribution is a function of all the resistances.

An actual joint between more than two strands is represented by a rather complicated lattice of resistances. Figure 10 shows the case of a joint between two three-strand cables put side by side and soldered.

Because of the differences between bridged resistances, even if all series resistances are theoretically equal, the center strands, 2 and $2^{\prime}$ of Fig. 10, carry more current (something like $10 \%$ to $20 \%$ more). A permutation of strands in the joints, from joint to joint, can help to equalize the currents. The oute: strand in the first joint becomes the inner strand in the second joint and so on, for example.

D. How to Make the Joints?

1. Although equal resistances are assumed at any similar position of a strand in the joint, an equal current distribution may not be achieved. If at each joint all the strands of one cable are soldered together and to another length of cable, some sort of trinsposition must be introdiced to equalize the currents. This can be done either by permuting the strands at every joint or locating the strands at every joint in some sort of transposed position.

2. In general, the series resistances are not equal. The best thing to do is probably to solder each strand of one length to one strand of the other length, independently, to keep all the strands insulated from each other along the eniire length of the cable in the coil. This causes an averaging of all the series resistances to occur, and the discrepancies between the current are reduc: d. Moreover, the currents or the overall resistances can be measured separately, and the series resistances can be adjusted by adding series resistances. This is probably the best way to equalize the currents when the number of strands is not too large. 

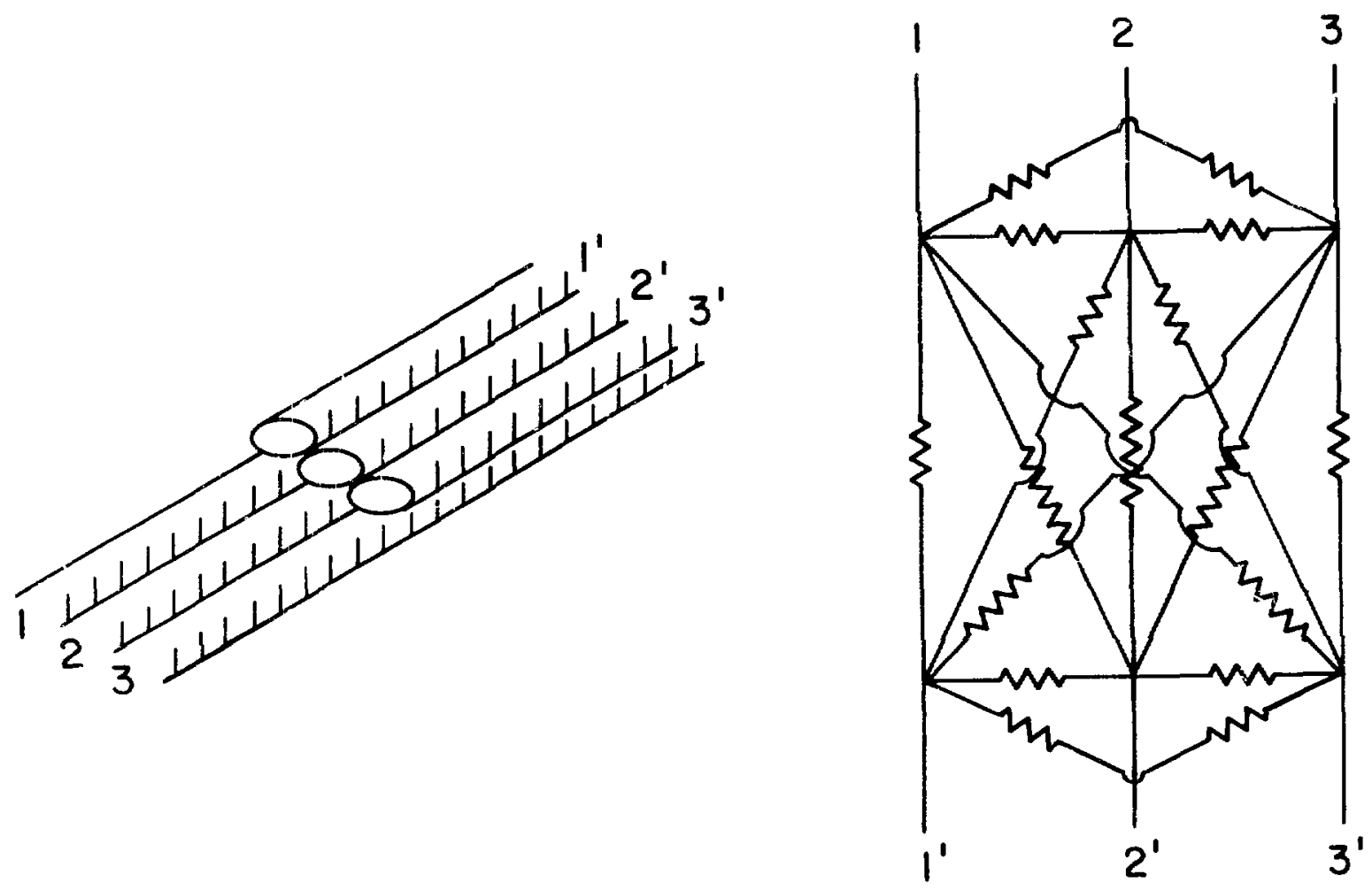

Fig. 10. A joint in a three-strand cable.

\section{REFERENCES}

1. A. Fevrier, I. Hlasnik, L. Kokavec, and J. P. Pouillange, Int. Report Saclay, SEDAP/73-124 (1973).

2. B. Turck, Cryogenics 14, 448 (1974).

3. P. Genevey, H. Nithart, J. L. Noel, Proceedings of the 5 th Int. Conf. Magnet Technology, Roma (Italy) 1975.

4. R. I. Schermer, B. Turck LASL Report (1979) Proceedings of the ICMC Conference Madison (August 1979). 